\title{
Radiation doses for mammography and its relationship with anthropo-technical parameters
}

\begin{abstract}
Background: Mammography is the radiographic examination of the breast. Radiation doses and cancer risks from breast imaging studies is under scrutiny hence, the need to investigate the dose received during mammography.

Objective of the study: To determine the doses received during mammography in two university teaching hospitals and to compare it with other established work.

Methodology: This study is a prospective cross- sectional study conducted in two university teaching hospitals in North eastern Nigeria. Sixty (60) patients were recruited for the study. Thermoluminiscent dosimeter (TLD) chips were exposed for cranio-caudal and medio-lateral examinations to record the entrance skin dose (ESD). TLD readings were obtained at the Centre for Energy Research and Training Zaria, Kaduna state, Nigeria. Dance formula was used to convert ESD to mean glandular dose (MGD). Student T-test was used to determine the relationship between the mean ESD obtained in the two centers and Pearson's correlation was used to determine the relationship between the MGD and anthropotechnical parameters. Statistical significance was set at $\mathrm{P}<0.05$.
\end{abstract}

Results: The total MGD for this study was $0.31 \pm 0.05 \mathrm{mGy}$ and $0.69 \pm 0.11 \mathrm{mGy}$ for cranio- caudal (CC) and medio-lateral oblique (MLO) respectively.

Conclusion: Mean Glandular doses were lesser than recommended standards. There was no statistical significant relationship $(p>0.05)$ between the MGD and anthropotechnical parameters.

Keywords: mammography, thermoluminiscent dosimeter, mean glandular dose, cranio-caudal view, medio-lateral oblique view
Volume 5 Issue I - 2018

\author{
Joseph Dlama Zira,' Christian \\ Chukwuemeka Nzotta ${ }^{2}$ \\ 'Department of Radiology, Abubakar Tafawa Balewa University \\ Teaching Hospital, Nigeria \\ ${ }^{2}$ Department of Radiography and Radiological Sciences, Nnamdi \\ Azikiwe University, Nigeria
}

\begin{abstract}
Correspondence: Joseph Dlama Zira, Department of Radiology, Abubakar Tafawa Balewa University Teaching Hospital, Bauchi, Nigeria, Tel +2348I3058272I,
\end{abstract}

Email josephdlama@gmail.com

Received: January 02, 2018 | Published: January 24, 2018
Abbreviations: TLD, thermoluminiscent dosimeter; ESD, entrance skin dose; MGD, mean glandular dose; MLO, medio-lateral oblique; CC, cranio- caudal; ICRP, international commission on radiological protection; ESD, entrance surface dose; SPSS, statistical package for social sciences

\section{Introduction}

Mammography is an x-ray examination of the breast usually done for breast cancer screening and accompanied by further development of both invasive and non-invasive radiological technique used for establishing the diagnosis of palpable and non palpable lesions. ${ }^{1,2}$ The major projection for $\mathrm{x}$-ray mammography includes cranio-caudal and mediolateral obliques view. There are two types of patients on whom mammograms are performed: symptomatic women in the clinic and asymptomatic women in breast screening programmes. ${ }^{3,4} \mathrm{The}$ standard technique used for breast imaging are x-ray mammography and real time ultrasonography with Doppler interrogation, magnetic resonance imaging, scintimammography and digital mammography. ${ }^{5}$ High quality mammography requires good and functional equipment with highly skilled and well trained radiographers. ${ }^{6}$ Breast cancer causes almost half a million deaths in the world per year but early detection has been demonstrated to reduce mortality by up to $30 \% .^{7-9}$

Measuring radiation dose to the breast has been performed using a variety of approaches including air kerma, entrance skin dose, mid- breast dose, total energy transmitted to the breast and the mean glandular dose. ${ }^{6,7}$ The average dose absorbed by the glandular tissue was found to be the most effective way of measuring absorbed dose of the breast because the mammary glands are most sensitive to ionizing radiation and to have the highest risk of developing radiation induced carcinogenesis..$^{8-10}$ Mean glandular dose (MGD) is the recommended metric used by many authorities such as International Commission on Radiological Protection (ICRP), United States National Council on Radiation Protection and Measurements, the British Institute of Physics and Engineering in Medicine (IPEM), the European Council Protocol and International Atomic Energy Agency (IAEA). ${ }^{11-13}$

Dose to glandular tissue of the breast cannot be directly measured using x-ray examination but can be assessed with certain standard assumptions that depend on breast characteristics and $\mathrm{x}$-ray spectra. ${ }^{6,10}$ MGD represents the effective dose absorbed by the breast and it is calculated from conversion factors that has been established, such factors relates MGD to entrance surface dose and allows for a wide and flexible range of $\mathrm{x}$-ray spectra, breast thickness and glandularity. ${ }^{13,14}$

The aim of the study is to determine the relationship between doses received during mammography and antropo-technical parameters in two university teaching hospitals and to compare it with other established work.

\section{Materials and methods}

\section{Methods}

The study is prospective cross sectional study carried out in Radiology departments of two University Teaching Hospitals located 
in North Eastern part of Nigeria. Sixty (60) patients were recruited for the study. The data in this study were collected from October 2015 to January 2016. The centers were chosen because they met the eligibility criteria for the study; having all the imaging modalities for the study and Nigerian Nuclear Regulatory Authority's Requirement for Authorization and Practice (Licensing) involving ionizing radiation.

\section{Machine specifications}

Mammography machine: for hospital A the machine is manufactured by Planmed OY, Helsinki Finland in April 2008 while that of hospital B was manufactured by Halogic Inco-operation USA in July 2012. There $\mathrm{kVp}$ and $\mathrm{mAs}$ range are 20-35 and 10-500 for hospital A and 20-40 and 10-400 for hospital B respectively. The inherent filtration for both hospitals was $30 \mu \mathrm{M}$ Molybdenum, $0.5 \mathrm{mmAl}, 25 \mu \mathrm{M}$ Rhodium

\section{Procedure}

Medio lateral oblique (MLO) and cranio-caudal (CC) views were considered. The patient breast was positioned on the support paddle, compression is then applied the machines uses automatic exposure control, it therefore provides the exposure factors to be used automatically namely $\mathrm{kVp}, \mathrm{mAs}$, anode/filter combination according to the breast granularity and thickness. The machine also provides the compressed breast thickness before exposure is made. The parameters were recorded for each patient and the compressed breast thickness is measured using flexible meter rule. Thermoluminiscent dosimeters (TLDs) were placed at the upper inner quadrant of the breast before any compression was made for both the CC and MLO views of both breasts. Two (2) TLDs were exposed for each patient. The exposed TLDs were labeled for proper identification and kept in black nylon away from radiation.

\section{Ethical clearance}

In line with Helsinki declaration (1964), ethical approval was obtained from the research ethics committee of the Faculty of Health Science and Technology, Nnamdi Azikiwe University Nnewi Campus and from each hospital under study. Informed consent form interpreted in Hausa language was filled by each (volunteer, Patient) participant in compliance with the Human Research Ethics Guidelines for patients who do not understand English Language. The first author/researcher also underwent web based training by National Health Institute on Research Ethics United States involving human subject for adequate knowledge on research procedures and guidelines involving human subjects.

\section{Dose determination for mammography}

After the TLDs were read by the TLD reader, the value gotten by the control TLD reading was subtracted from the value of the actual TLD to get the value of the Entrance Surface Dose (ESD).

To get the Mean glandular Dose the conversion factors derived by Dance et al. ${ }^{15}$ was used to calculate the MGD. ${ }^{15}$
The MGD was calculated using this formula:

$$
M G D=K \times g \times C \times s
$$

Where K Entrance Surface Dose.

$\mathrm{g}=\mathrm{ESD}$ to MGD conversion factor on the assumption that the entire breast has a glandularity $50 \%$.

$\mathrm{C}=$ Conversion factor for difference in breast composition other than $50 \%$ glandularity.

$\mathrm{s}=\mathrm{is}$ the conversion factor for different $\mathrm{x}$-ray spectrum which can be due to different anode/filter combination e.g. $\mathrm{Mo} / \mathrm{Mo}, \mathrm{Mo} / \mathrm{Rh}$.

\section{Data analysis}

Data was obtained and saved on a computer Microsoft excel spread sheet and categorized for each examination and imaging modality respectively. It was independently checked by a statistician and two senior radiographers. Statistical Package for Social Sciences (SPSS) version 21.0 was used to analyze the mean and standard deviation of the anthropometric variables, technical parameters and radiation dose received. Using Kolmogorov- Smirnov to test for normality of data distribution it was verified that, for $95 \%$ of confidence level, there was a normal distribution. Therefore, we used a parametric test, suitable for the set of data and analysis. Pearson's correlation was used to determine the relationship between radiation dose and compressed breast thickness while students t-test was used to compare the MGD for the two hospitals. Statistical significance was set at $\mathrm{p}<0.05$.

\section{Results}

Table 1 shows mean and standard deviation of the entrance skin dose, mean glandular dose and diagnostic reference levels for mammography examination. The mean entrance skin dose for cranio-caudal and Medio lateral oblique are $0.50 \pm 9.48 \mathrm{mGy}$ and $0.70 \pm 0.74 \mathrm{mGy}$ for Hospital A, $0.31 \pm 0.05 \mathrm{mGy}$ and $0.69 \pm 0.11 \mathrm{mGy}$ for hospital B. The total mean and standard deviation for both hospitals were $0.48 \pm 0.69 \mathrm{mGy}$ and $0.68 \pm 0.40 \mathrm{mGy}$ for cranio-caudal and Medio lateral oblique respectively. The mean glandular dose for craniocaudal and Medio lateral oblique are $0.31 \pm 0.05$ and $0.69 \pm 0.11 \mathrm{mGy}$ (Table 2 \& Table 3 )

Table 4 shows the T-test comparism of radiation dose and some technical parameters for mammography examination between hospital A and B. Detail result from the table shows that when the mean doses for the hospitals were compared there was no statistical significant relationship $(\mathrm{p}>0.05)$ between $\mathrm{mAs}, \mathrm{kVp}$ and mean glandular dose for the hospitals.

Table 5 shows comparism of Mean Glandular Dose for mammography examination with reference values for European commission, United Kingdom and Australia. The reference values for Australian radiation protection and nuclear safety agency (ARPANSA), EC, UK and this work were $0.88 \mathrm{mGy}, 2.0 \mathrm{mGy}, 2.0 \mathrm{mGy}$ and $0.31 \mathrm{mGy}$ for cranio-caudal view and $1.30 \mathrm{mGy}, 2.0 \mathrm{mGy}, 2.1 \mathrm{mGy}$ and $0.69 \mathrm{mGy}$ for mediolateral- oblique.

Table I Mean glandular dose (MGD) received for mammography examination

\begin{tabular}{lllll}
\hline Examination & $\begin{array}{l}\text { Mean ESD }(m G y) \text { hospital } \\
\text { A }\end{array}$ & $\begin{array}{l}\text { Mean ESD(mGy) hospital } \\
\text { B }\end{array}$ & $\begin{array}{l}\text { Mean ESD(mGy) } \\
\text { both }\end{array}$ & MGD(mGy) \\
\hline Cranio-caudal & $0.50 \pm 0.48$ & $0.31 \pm 0.05$ & $0.48 \pm 0.69$ & $0.31 \pm 0.05$ \\
Medio lateral oblique & $0.70 \pm 0.74$ & $0.69 \pm 0.11$ & $0.68 \pm 0.40$ & $0.69 \pm 0.11$
\end{tabular}

ESD, entrance skin dose; MGD, mean glandular dose 
Table 2 The relationship between doses received by patients and anthropometric parameters for mammography examination. There was no statistical significant relationship ( $p>0.05$ ) between the dose and compressed breast thickness, weight, height and BMI

\begin{tabular}{|c|c|c|c|c|}
\hline Dose versus & CBT(cm) & Weight(kg) & Height $\left(\mathrm{m}^{2}\right)$ & BMI $\left(\mathrm{kg} / \mathrm{m}^{2}\right)$ \\
\hline Examination & $r p$ & $r p$ & $r p$ & $r p$ \\
\hline Cranio caudal & $0.134,0.479$ & $-0.197,0.297$ & $0.255,0.174$ & $-0.220,0.242$ \\
\hline Medio lateral oblique & $-0.197,0.297$ & $-0.219,0.244$ & $0.324,0077$ & $-0.352,0.057$ \\
\hline
\end{tabular}

**, Correlation is significant at the 0.01 level (2-tailed), *, Correlation is significant at the 0.05 level (2-tailed)

CBT, compressed breast thickness

Table 3 Relationship between doses received by patients during radiographic examination and technical parameters

\begin{tabular}{llll}
\hline \multirow{2}{*}{ Examination } & \multirow{2}{*}{ Technical parameters } & \multicolumn{2}{c}{ MGD vs technical parameters } \\
\cline { 3 - 4 } & & R-value & p-value \\
\hline Cranio-caudal view mammography & $\mathrm{kVD}$ & 0.003 & 0.989 \\
& $\mathrm{mAs}$ & 0.139 & 0.464 \\
Mammagraphy & $\mathrm{FSD}$ & $-0.08 \mathrm{0}$ & 0.669 \\
medio lateral oblique & $\mathrm{kVp}$ & 0.012 & 0.021 \\
& $\mathrm{mAs}$ & -0.199 & 0.292
\end{tabular}

**, Correlation is significant at the 0.01 level (2-tailed), *, Correlation is significant at the 0.05 level (2-tailed)

Table 4 Comparison of patient's mean glandular dose and technical parameters for mammography examination between Hospital A and Hospital B

\begin{tabular}{|c|c|c|c|c|c|}
\hline Examination & Parameters & Mean \pm Std (Hospital A) & Mean士Std (Hospital B) & P-value & T-value \\
\hline \multirow[t]{3}{*}{ Cranio Caudal View } & $\mathrm{mAs}$ & $80.17 \pm 10.00$ & $80.53 \pm 5.00$ & $p>0.05$ & 0.056 \\
\hline & $k V_{p}$ & $21.17 \pm 10.00$ & $20.50 \pm 4.00$ & $p>0.05$ & 0.108 \\
\hline & MGD & $0.3 I \pm 0.20$ & $0.50 \pm 0.10$ & $p>0.05$ & 1.472 \\
\hline \multirow[t]{3}{*}{ Medio Lateral Oblique (MLO) } & $\mathrm{mAs}$ & $80.20 \pm 10.00$ & $80.20 \pm 2.00$ & $p>0.05$ & 0.000 \\
\hline & $k V_{p}$ & $21.03 \pm 10.00$ & $20.23 \pm 5.00$ & $p>0.05$ & 0.124 \\
\hline & MGD & $0.69 \pm 0.10$ & $0.73 \pm 015$ & $p>0.05$ & $0.4 I I$ \\
\hline
\end{tabular}

*, Significant at $\mathrm{P}<0.05$ when compared between Hospital $\mathrm{A}$ and Hospital $\mathrm{B}$ variables

MGD, mean glandular dose; $k V_{p}$, kilo volt peak; $m A s$, milli ampere seconds

Table 5 Comparism of DRLs for mammography in this work with European Commission, United Kingdom and Australian radiation protection and nuclear safety agency DRLs

\begin{tabular}{lllll}
\hline Examination & ARPANSA DRL(mGy) & EC DRL(mGy) & UK DRL(mGy) & MGD(mGy) \\
\hline Cranio-caudal & 0.88 & 2.0 & 2.0 & $0.31 \pm 0.05$ \\
Medio lateral oblique & 1.30 & 2.0 & 2.1 & $0.69 \pm 0.11$ \\
\hline
\end{tabular}

EC, european commission; UK, united kingdom

ARPANSA, Australian radiation protection and nuclear safety agency

\section{Discussion}

The study determined the relationship between doses received during mammography and antropo-technical parameters in two university teaching hospitals and to compare it with other established work. The hospitals studied were divided into two A and B respectively. There are three university teaching hospitals in North Eastern Nigeria as at the time of the study. However, hospitals A and B were chosen because they met the inclusion criteria for the study having the necessary functional imaging facility (mammography). A total of sixty patients participated in this study.
Table 1 shows mean and standard deviation of the entrance skin dose and mean glandular dose for mammography examination. The mean entrance skin dose for cranio-caudal and medio lateral oblique are $0.50 \pm 9.48 \mathrm{mGy}$ and $0.70 \pm 0.74 \mathrm{mGy}$ for Hospital A, $0.31 \pm 0.05 \mathrm{mGy}$ and $0.69 \pm 0.11 \mathrm{mGy}$ for hospital B. The total mean and standard deviation for both hospitals were $0.48 \pm 0.69 \mathrm{mGy}$ and $0.68 \pm 0.40 \mathrm{mGy}$ for cranio-caudal and Medio lateral oblique respectively. The mean glandular dose for cranio-caudal and Medio lateral oblique are $0.31 \pm 0.05$ and $0.69 \pm 0.11 \mathrm{mGy}$. The diagnostic reference level for cranio-caudal and Medio lateral oblique are $0.63 \mathrm{mGy}$ and $1.04 \mathrm{mGy}$. The use of different breast imaging technique in patient's 
dose management is increasing due to technology advancement availability of radiological equipment and health care cost cutting measures. ${ }^{1,17}$ The mean dose in general mammography examination was found to be larger than the values reported in the studies done in India and Sudan. In the study, the mean number of runs and images per examination category were comparable. ${ }^{2}$ The uniformity trend in radiographic imaging technique for most examination in this study is also supportive for the potential for standardization of anatomical related imaging techniques and protocols.

The results of the MGD estimated from this study shows that dose from mammography in this study is lower than the result gotten from another work on the mean glandular doses for woman undergoing mammography breast screening in Oyo State, Nigeria. The value gotten from his work was $0.26-2.26 \mathrm{mGy}$ for the MLO Views and 0.08 to $5.30 \mathrm{mGy}$. The difference can be due to difference in tube output and the use of film screen combination of which some Centre were using digital mammography. However this study agrees with this study which discovered that over 90 of patients had MGD values less than $2.5 \mathrm{mGy}$ which is below the guidance level of $3 \mathrm{mGy}$. The value of MGD gotten from the work is also significantly lower than the one gotten from a study that calculates the MGD assessment for phantoms' and patients in which the phantom gave the MGD of $1.9 \mathrm{mGy}$. When MGD is supplemented by a patient dose survey, the average MGD per image was $2.8 \mathrm{mGy}$ for CC and $4.3 \mathrm{mGy}$ for the MLO. Differences may be due to differences in tube output and breast granularity.

Table 2 shows the relationship between doses received by patients and anthropometric parameters for mammography examination. There was no statistical significant relationship $(p>0.05)$ between the dose and compressed breast thickness, weight, height and BMI.

Table 3 shows the relationship between doses received by patients and anthropometric parameters for computed tomography examination. There was no statistical significant $(\mathrm{p}>0.05)$ relationship between computed tomography dose index with thickness, weight, and height BMI head CT and abdominal CT. However, Chest CT show statistical significant relationship $(\mathrm{p}<0.05)$ with weight and height. Table $4 \&$ Table $4 \mathrm{a}$, shows the relationship between doses received by patients during radiological examination and technical parameters. The result indicated that when the mean dose of entrance skin dose (ESD) and technical variables (FSD, KVp and mAs) of various radiological examinations for chest $\mathrm{x}$-ray $\mathrm{PA} / \mathrm{AP}$, chest $\mathrm{x}$-ray lateral, hand dorsi palmar, abdominal x-ray, pelvic x-ray, hand dorsi, hand dorsi palmar oblique, cranio caudal view, medio lateral oblique (MLO), dental x-ray, when correlated they all showed no statistical significant differences $(\mathrm{P}>0.05)$, but in $\mathrm{PA}$ chest $\mathrm{x}$-ray there was a positive significant correlation $(\mathrm{P}<0.05)$ between focus to skin distance (FSD) and entrance skin dose (ESD), while $\mathrm{kVp}$ relationship with Entrance skin dose (ESD), showed a negative significant correlation $(\mathrm{P}<0.05)$ as shown in the Table 1. Although, breast thickness is not the only factor to have an effect on mean glandular dose, it is the most consistently reported. Other factors that affect MGD are not consistently reported. ${ }^{6,7}$ Other factors reported include $\mathrm{kVp}$, target filter combination, HVL, mAs 6. The lack of documented protocol and etiquette in establishing DRLs in Nigeria and other countries makes it difficult to come up with a guideline and recommendations on DRL for mammography. ${ }^{6,15}$

Table 4 shows the T-test comparism of radiation dose and some technical parameters for mammography examination between hospital
A and B. Detail result from the table shows that when the mean doses for the hospitals were compared there was no statistical significant relationship ( $p>0.05$ ) between $\mathrm{mAs}, \mathrm{kVp}$ and mean glandular dose for the hospitals. This corroborates with another study done by Lourenco et al. 2013, sponsored by European society of Radiologist which indicated that there is no statistical significant relationship between two hospitals $(\mathrm{p}=0.090)$.

Table 5 shows comparism of established reference levels for mammography with that of European commission, United Kingdom and Australia. The reference values for Australian radiation protection and nuclear safety agency (ARPANSA), EC, UK and this work were $0.88 \mathrm{mGy}, 2.0 \mathrm{mGy}, 2.0 \mathrm{mGy}$ and $0.63 \mathrm{mGy}$ for cranio-caudal view. DRLs for mammography have been established across the world, and variable methods and techniques were used. However, an internationally accepted protocol that includes dose measurement method, conversion factor compressed breast thickness for patients or phantoms and DRL percentile needs to be established before important useful and accurate international comparison can be made. ${ }^{6}$

\section{Recommendations}

I. The research work shows that there is need to optimize operations in hospitals in North eastern Nigeria and probably in most hospitals in Nigeria. The optimization step may start with the regulatory body mandating radiographers to take part in various refreshers and update course for them to be aware of the current trends and recent developments on how to properly and effectively dispense radiation in diagnosis.

II. The hospitals should implement a functional and standing radiation safety committee appoint a radiation safety adviser and radiation safety officer that will be trained by the regulatory body on radiation safety. The essence of enacting this committee is to saddle them with the responsibility of monitoring the staff, developing a facility patient dose database that will be used to evaluate radiation dose whenever the need arises and ensuring radiation safety culture in radiation practices.

III. This kind of study should be conducted in all the regions of Nigeria so that Nigeria can successfully have a National DRLs for radiological examination. It is therefore suggested that the Nigerian Nuclear Regulatory Authority should collaborate with academicians and clinical researchers to come up with regional and national DRLs and should also come up with a policy for periodic review after every fiveyears as a DRLs guidelines and publish it as a regulatory guideline in ionizing radiation regulations.

IV. A culture of regular dose measurement, quarterly quality control, film reject analysis, image quality assessment should be inculcated in each facility as recommended by IAEA as a main part of diagnostic radiology procedures and installations.

V. Nuclear regulatory Authority should come up with DRLs document and guidelines for Nigeria by having a sample from representative Geo- political zones in Nigeria. This study suggests that a committee should be constituted and mandated to Set DRLs for Nigeria and it should be revisited after every fiveyears. This committee should comprise of academic researchers, clinical researchers, radiographers, radiologist, medical physicist and engineers. 


\section{Conclusion}

This present determined mean glandular dose (MGD) values for mammography in two major referral hospitals in North Eastern Nigeria with larger patient throughput. The MGD values for mammography in this study are lower compared to that of ARPANSA, UK and European Commission. This study has an educational and regulatory function to the radiology community and furthermore provides a benchmark to assist any statutory organization to establish DRLs for diagnostic radiology practices in Nigeria, Africa and the world entirely.

\section{Acknowledgements}

We acknowledge all the Radiographers, Radiologist and medical physicist in all the hospitals studied.

\section{Conflict of interest}

Author declares that there is no conflict of interest.

\section{References}

1. Joseph Z, Nzotta CC. The need to establish dose reference levels for radiological examinations in Nigeria: Radiographers role. Nigerian Journal of Medical Imaging and Radiation Therapy. 2016;5(1):25-39.

2. Abdullahi M, Shittu H, Arabisala A, et al. Diagnostic Reference Level for Adult Brain Computed Tomography Scans: A Case Study of a Tertiary Health Care Center in Nigeria. Journal of Dental and Medical Sciences (IOSR-JDMS). 2015;1(2):66-75.

3. IAEA. Radiological Protection for Medical Exposure to Ionizing Radiation. International Atomic Energy Agency. Vienna; 2002.

4. Joseph D, Obetta C, Nkubli F, et al. Rationale for implementing dose reference levels as a quality assurance tool in medical radiography in Nigeria. IOSR Journal of dental and medical sciences. 2014;13(12):41-45.

5. Yousif M, Ali H, Suha M. Assessment of mean glandular dose (MGD) received in mammography examination in Khartoum. International journal of advanced research. 2016;4(1):198-203.
6. Moayyad ES, Patrick Brennan C, Mark F Mc Entee. Diagnostic Reference Levels in Digital Mammography: A systematic review. Radiat Prot Dosimetry. 2014;164(4):608-619.

7. Wall BF, Shrimpton PC. Patient dose Protocol and Trends in UK. Radiation Protection Dosimetry. 1995;57:359-362.

8. Wallace AB. The Implementation of Diagnostic Reference Levels to Australian Radiology Practice. J Med Imaging Radiat Oncol. 2010;54(5):465-471.

9. ICRP. Recommendation of International Commission on Radiological Protection. Oxford: ICRP Publication 64 Pergamum Press; Ann ICRP. 2000:21(1-3).

10. ICRP. Diagnostic reference levels in medical imaging: Review and additional advice. Ann ICRP. 2001;31(4):33-35.

11. ARPANSA. Code of Practice for Radiation Protection in Medical Applications of Ionizing Radiation. National Diagnostic Reference levels Fact sheet. Yallambie: A publication of Australian Radiation Protection and Nuclear Safety Agency. 2014.

12. ARPANSA. Code of Practice for Radiation Protection in Medical Applications of Ionizing Radiation. National Diagnostic Reference levels Fact sheet. A publication of Australian Radiation Protection and Nuclear Safety Agency. Yallambie; 2008.

13. IAEA. International Basic Safety Standards for Protection against Ionizing Radiation and for safety of radiation sources IAEA Safety Series No. 115-1. International Atomic Energy Agency. Vienna Austria; 1996.

14. Recommended standard for routine performance testing of diagnostic $x$-ray imaging system. IPEM Report 91 . York, Institute of physics and engineering in medicine, UK; 2015.

15. Dance DR, Young KC, Van Eugen RE. Further factors for the estimation of mean glandular dose using the united kingdom, European and IAEA breast dosimetry protocols. Phys Med Biol. 2009;54(14):4361-4372.

16. European Protocol on Dosimetry in Mammography. CEC. 1996.

17. Ogunseyinde AO, Adeniran Sam, Obed RI, et al. Comparison of entrance skin dose of some $\mathrm{x}$-ray examinations with CEC reference doses. Journal of Radiation Protection and dosimetry. 2002;98(2):231-234. 